\title{
Natural convection in a rotating cavity partially filled with a porous medium under the effect of a local heater
}

\author{
Stepan Mikhailenko, and Mikhail Sheremet* \\ Laboratory on Convective Heat and Mass Transfer, Tomsk State University, 36 Lenin Avenue, 634050 Tomsk, Russia
}

\begin{abstract}
The combined effect of uniform rotation and porous layer on natural convective heat transfer in a square cavity with a local heater has been studied numerically. The local thermal equilibrium model with the Brinkman-extended Darcy law has been used for description of the heat transfer and fluid flow within the porous layer. Governing equations with corresponding boundary conditions formulated using the nondimensional stream function and vorticity variables have been solved by the finite difference method. The effects of Taylor number and porous layer thickness on fluid flow and heat transfer have been examined. It has been found a non-linear effect of the porous layer thickness on the heat transfer rate.
\end{abstract}

\section{Introduction}

Rotating cavities filled with porous material can be met in different engineering applications including heat exchangers, solar collectors, chemical reactors and others. Numerical and experimental analysis of convective heat transfer within rotating cavities has been performed by different authors [1-6]. Thus, Zhao et al. [1] have numerically analyzed natural convection in a fluid saturated porous horizontal cylinder under the effect of rotation about its axis. They have found that rotation significantly decreases the overall heat transfer. Jin et al. [2] have studied experimentally and numerically the heat transfer performance from discrete heat sources in a rotating cavity. It has been revealed that due to thermal inertia, rotation leads to a steady temperature field inside the solid zone and unsteady flow and temperature fields within the fluid zone, even in the low rotation speed range. Linear stability of a rotating fluid-saturated porous layer heated from below and cooled from above has been investigated by Malashetty et al. [3]. The obtained results have shown the competition between the processes of rotation and thermal diffusion that causes the convective instability to set in an oscillatory mode rather than stationary. The influence of local thermal non-equilibrium conditions on the linear and non-linear thermal instability in a nanofluid saturated rotating porous layer has been examined by Agarwal and Bhadauria [4]. They found that the system tends to be stabilized by the presence of rotational component in the porous medium and the onset of convection is delayed. The influence of rotation on the onset of convection in an anisotropic horizontal porous layer with internal heat source has been analyzed by Bhadauria et al. [5].

The objective of the present work is to analyze numerically natural convection of water inside a rotating square cavity partially filled with a porous material under the effect of local heater of constant temperature using local thermal equilibrium conditions. We believe that this work is noteworthy, and its usefulness for different engineering systems as discussed above motivated us.

\section{Analysis and modelling}

A square cavity with walls of length $H$ with two opposite isothermally cold walls $(\bar{x}=0, \bar{x}=H)$ rotates counterclockwise with a constant angular velocity $\omega_{0}$ (see Fig. 1). A heater of constant high temperature is located on the wall $\bar{y}=0$. A homogeneous porous layer covers the heated element. The following assumptions are made in the study:

- the Brinkman-extended Darcy model is used for the porous layer;

- the Boussinesq approximation is applied for description of the buoyancy force effect;

- the fluid phase and the solid matrix of the porous medium are described using the local thermal equilibrium conditions;

- the interface between the clear fluid and porous medium is permeable and fluid can penetrates through this border;

- properties of the fluid and porous medium are homogeneous and isotropic;

- boundaries of the cavity are assumed to be impermeable.

The equations governing the unsteady Brinkman natural convection flow and heat transfer inside the rotating porous cavity using the above mentioned assumptions can be written in the form including the the local thermal equilibruim inside the porous layer.

\footnotetext{
*Corresponding author: Michael-sher@yandex.ru
} 


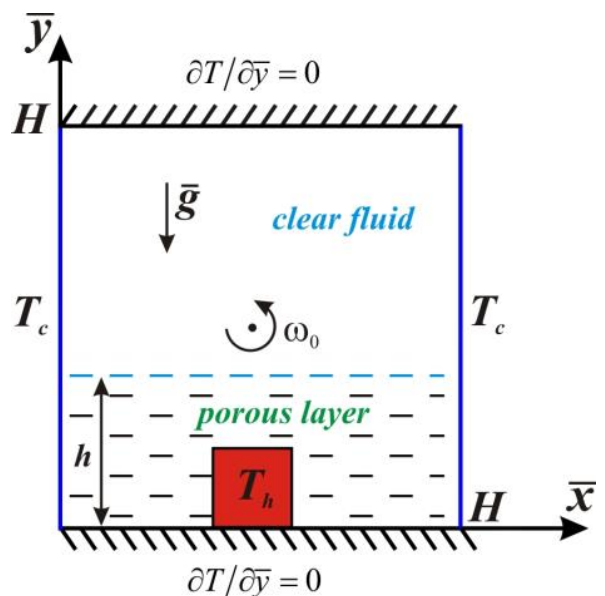

Fig. 1. Physical model and coordinate system.

Taking into account the dimensionless stream function $(\psi)$ and vorticity $(\omega)$ variables as well as above-mentioned approaches, the governing equations can be formulated in the following form:

- for the clear fluid layer [7]:

$$
\begin{gathered}
\frac{\partial^{2} \psi}{\partial x^{2}}+\frac{\partial^{2} \psi}{\partial y^{2}}=-\omega \\
\frac{\partial \omega}{\partial \tau}+u \frac{\partial \omega}{\partial x}+v \frac{\partial \omega}{\partial y}=\frac{1}{\sqrt{T a}}\left(\frac{\partial^{2} \omega}{\partial x^{2}}+\frac{\partial^{2} \omega}{\partial y^{2}}\right)+ \\
+\frac{R a}{\operatorname{Pr} \cdot T a}\left[\frac{\partial \theta}{\partial x} \cos (\tau)-\frac{\partial \theta}{\partial y} \sin (\tau)\right] \\
\frac{\partial \theta}{\partial \tau}+u \frac{\partial \theta}{\partial x}+v \frac{\partial \theta}{\partial y}=\frac{1}{\operatorname{Pr} \cdot \sqrt{T a}}\left(\frac{\partial^{2} \theta}{\partial x^{2}}+\frac{\partial^{2} \theta}{\partial y^{2}}\right)
\end{gathered}
$$

- for the porous layer [8]:

$$
\begin{gathered}
\frac{\partial^{2} \psi}{\partial x^{2}}+\frac{\partial^{2} \psi}{\partial y^{2}}=-\omega \\
\varepsilon \frac{\partial \omega}{\partial \tau}+u \frac{\partial \omega}{\partial x}+v \frac{\partial \omega}{\partial y}=\frac{\varepsilon}{\sqrt{T a}}\left(\frac{\partial^{2} \omega}{\partial x^{2}}+\frac{\partial^{2} \omega}{\partial y^{2}}-\varepsilon \frac{\omega}{D a}\right)+ \\
+\frac{\varepsilon^{2} \cdot \operatorname{Ra}}{\operatorname{Pr} \cdot T a}\left[\frac{\partial \theta}{\partial x} \cos (\tau)-\frac{\partial \theta}{\partial y} \sin (\tau)\right] \\
\eta \frac{\partial \theta}{\partial \tau}+u \frac{\partial \theta}{\partial x}+v \frac{\partial \theta}{\partial y}=\frac{\alpha_{p m} / \alpha_{f}}{\operatorname{Pr} \cdot \sqrt{T a}}\left(\frac{\partial^{2} \theta}{\partial x^{2}}+\frac{\partial^{2} \theta}{\partial y^{2}}\right)
\end{gathered}
$$

Following the geometry in Fig. 1, we assume that the initial and boundary conditions for these equations are $\psi=0, \omega=0, \theta=0$ at $\tau=0$

$\psi=0, \omega=-\frac{\partial^{2} \psi}{\partial x^{2}}, \theta=0$ at $x=0,1$

$\psi=0, \omega=-\frac{\partial^{2} \psi}{\partial y^{2}}, \frac{\partial \theta}{\partial y}=0$ at $y=0,1$

$\psi=0, \omega=-\frac{\partial^{2} \psi}{\partial n^{2}}, \theta=1$ at the heater surface

$$
\begin{aligned}
& \left\{\begin{array}{l}
\left.\psi\right|_{p m}=\left.\psi\right|_{f} \\
\left.\frac{\partial \psi}{\partial y}\right|_{p m}=\left.\frac{\partial \psi}{\partial y}\right|_{f} \quad\left\{\left.\frac{\partial \omega}{\partial y}\right|_{p m}=\left.\frac{\partial \omega}{\partial y}\right|_{f}=\left.\omega\right|_{f}\right.
\end{array}\right. \\
& \left\{\begin{array}{l}
\left.\theta\right|_{p m}=\left.\theta\right|_{f} \\
\left.\frac{k_{p m}}{k_{f}} \frac{\partial \theta}{\partial y}\right|_{p m}=\left.\frac{\partial \theta}{\partial y}\right|_{f}
\end{array}\right. \text { at the fluid-porous interface }
\end{aligned}
$$

Here $R a$ is the Rayleigh number, $P r$ is the Prandtl number, $T a$ is the Taylor number and $D a$ is the Darcy number, which are defined as

$$
\begin{aligned}
& R a=\frac{\rho g \beta \Delta T H^{3}}{\mu \alpha}, \quad \operatorname{Pr}=\frac{\mu}{\rho \alpha}, \\
& D a=\frac{K}{H^{2}}, \quad T a=\frac{\rho^{2} \omega_{0}^{2} H^{4}}{\mu^{2}}
\end{aligned}
$$

The physical quantity of interest is the average Nusselt number at the heater surface, which can be defined as

$$
\overline{N u}=-\frac{1}{l} \int_{0}^{l} \frac{\partial \theta}{\partial \mathbf{n}} d \zeta
$$

Equations (1)-(6) with corresponding initial and boundary conditions were solved using an in-house computational code developed by means of the finite difference method of the second order accuracy [7-9]. The developed mathematical model and numerical technique have been verified comprehensively using the data of other authors [7-9].

The grid independency test has been performed for the present formulation (see Fig. 1) at $R a=10^{5}, T a=10^{4}$, $D a=10^{-3}, \operatorname{Pr}=6.82, \varepsilon=0.398, \gamma=h / H=0.5$. Three uniform grids are tested. Figure 2 presents the effect of the grid parameters on the profiles of temperature (Fig. $2 a$ ) and vertical velocity (Fig. $2 b$ ) at cross-section $y=0.5$ and $\phi=0$ or $2 \pi$.
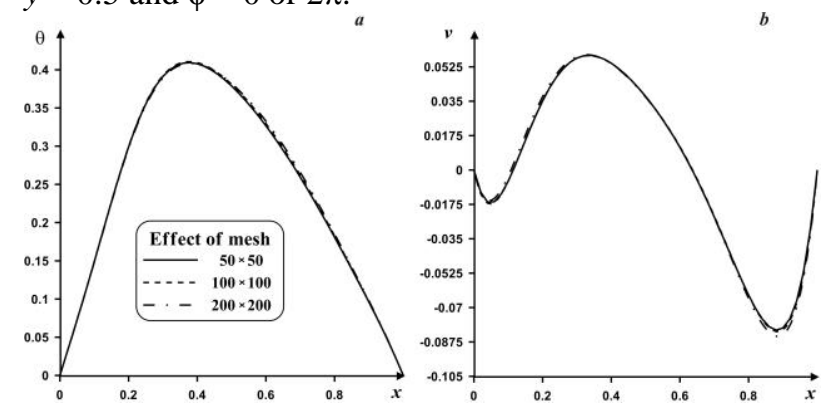

Fig. 2. Variations of the temperature $(a)$ and vertical velocity (b) along the cross-section $y=0.5$ versus the mesh parameters.

Using the results presented in Fig. 2, the uniform grid of $100 \times 100$ points has been chosen for the present study.

\section{Results and Discussion}

Analysis has been carried out for the following values of the governing parameters: Rayleigh number $\left(R a=10^{5}\right)$, Taylor number $\left(\mathrm{Ta}=10^{3}-10^{6}\right)$, Prandtl number $(\operatorname{Pr}=6.82)$, Darcy number $\left(D a=10^{-3}\right)$, porosity $(\varepsilon=0.398)$, and porous layer height $(\gamma=0.25-0.75)$. Particular efforts have been focused on the effects of the 
Taylor number and porous layer height on streamlines and isotherms as well as average Nusselt number and fluid flow rate inside the cavity.

Figure 3 shows streamlines during complete revolution with an angle step $\pi / 2$ for $T a=10^{4}, \gamma=0.5$. It should be noted that the presented streamlines have been obtained for the steady state, when the following revolutions repeat the previous ones. For description of these isolines of stream function it is possible to analyze the evolution of convective vortices formed near the heater. In the case of $\phi=0$ one can find a development of small vortex from the left side of the heater and large vortex from the right side of the heater. A rotation of the cavity for $\phi=\pi / 2$ leads to a growth of the sizes of the left convective cell, while the right one decreases in sizes. The following rotation illustrates the development and attenuation of mentioned convective cells. It is interesting to note that major convective cell core is located inside the clear fluid layer, where circulation occurs without the resistance from the solid matrix of porous medium.

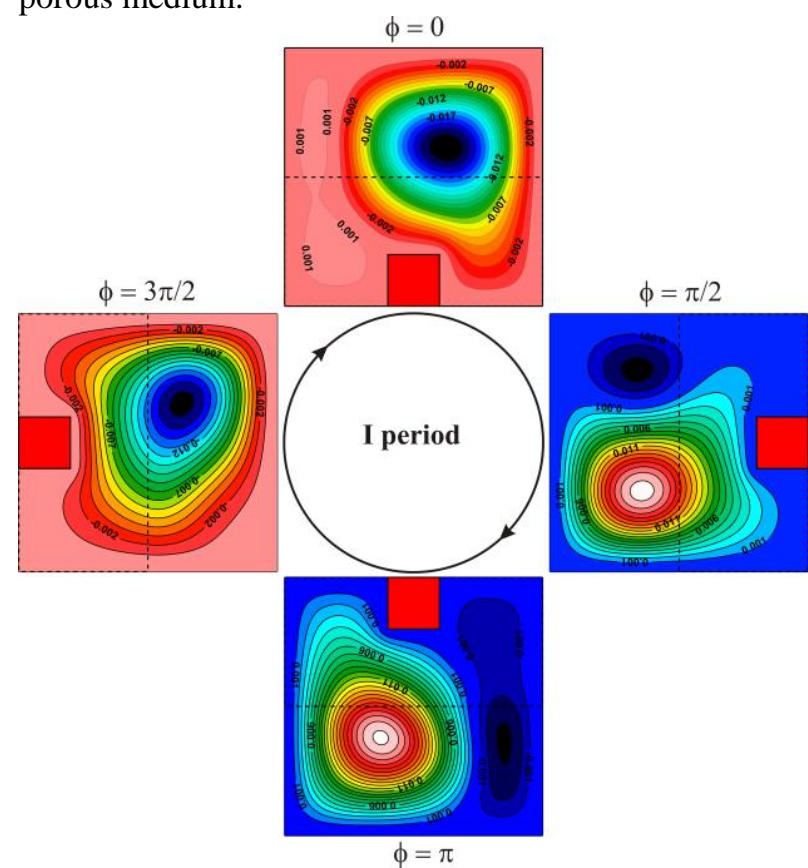

Fig. 3. Streamlines during complete revolution for $T a=10^{4}$, $\gamma=0.5$.

Figure 4 demonstrates the isotherms inside the cavity during the complete revolution in accordance to the mentioned streamlines. It is worth noting that major heat transfer inside the porous layer is a heat conduction, while inside the clear fluid one can find more essential distortion of isolines of temperature due to intensive convective circulation. It is well-known that rotation can lead to the convective flow attenuation. Such effect of rotation can be found in Fig. 5.

The effects of the Taylor number and porous layer thickness on the average Nusselt number at the heated element and fluid flow rate during one complete revolution are presented in Fig. 5. As has been mentioned above, high angular velocity illustrates an essential attenuation of convective flow and heat transfer. At the same time, for $T a=10^{6}$ oscillations for the average Nusselt number and fluid flow rate vanish, while in the case of low angular velocity $T a=10^{3}$ one can find intensive circulation and high heat transfer rate.

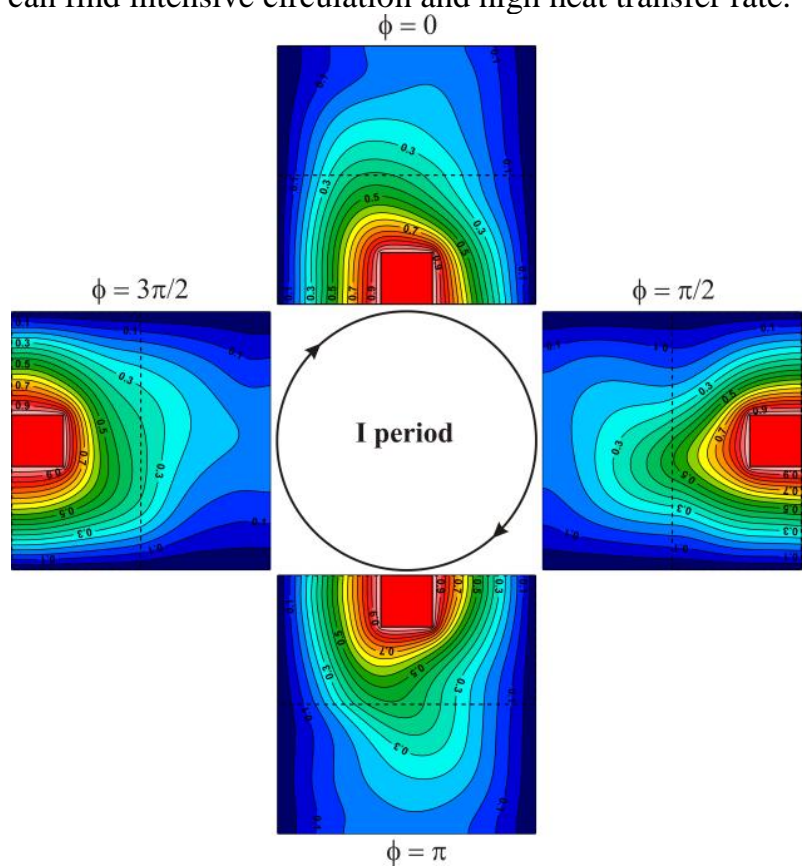

Fig. 4. Isotherms during complete revolution for $T a=10^{4}$, $\gamma=0.5$.

$a$
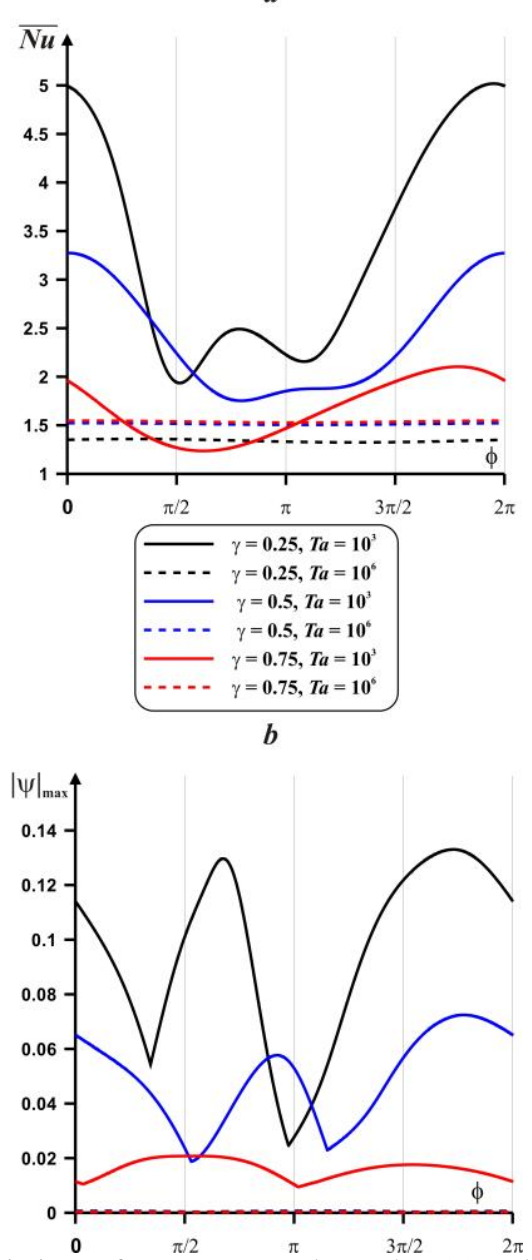

Fig. 5. Variations of average Nusselt number $(a)$ and fluid flow rate $(b)$ with rotation angle during one complete revolutions for different values of porous layer thickness and Taylor number. 
A growth of the porous layer thickness results in the reduction of the heat transfer rate in the case of low Taylor number, while in the case of high Taylor number a rise of $\delta$ leads to weak heat transfer enhancement. At the same time, high porous layer thickness illustrates low amplitude of the heat transfer rate oscillations. The same changes can be found in distributions of the fluid flow rate with $\phi$.

\section{Conclusions}

Natural convection in a partially porous square cavity with a local heater under the effect of uniform rotation has been studied numerically using the local thermal equilibrium approach. Particular efforts have been focused on the effects of the Taylor number and porous layer thickness on streamlines and isotherms as well as average Nusselt number at the heater surface and fluid flow rate. It has been found that in the case of steady state the complete revolution can be considered as an evolution of the convective cells near the heater. A growth of the Taylor number results in the convective flow and heat transfer attenuation. It should be noted that for low Taylor numbers a rise of $\gamma$ leads to the heat transfer rate reduction, while for high values of Taylor number a rise of $\gamma$ results in weak heat transfer enhancement.

\section{Acknowledgements}

This work was conducted as a government task of the Ministry of Education and Science of the Russian Federation (Project Number 13.9724.2017/8.9).

\section{References}

1. M. Zhao, L. Robillard, M. Prud'homme, Heat Mass Transfer 31 (1996).

2. L.F. Jin, K.W. Tou, C.P. Tso, Experimental Heat Transfer 18 (2005).

3. M.S. Malashetty, M. Swamy, S. Kulkarni, Physics of Fluids 19 (2007).

4. S. Agarwal, B.S. Bhadauria, Transport Porous Media 90 (2011).

5. B.S. Bhadauria, A. Kumar, J. Kumar, N.C. Sacheti, P. Chandran, Transport Porous Media 90 (2011).

6. A. Alhusseny, A. Turan, Heat Mass Transfer 51 (2015).

7. S.A. Mikhailenko, M.A. Sheremet, Numerical Heat Transfer A 72 (2017).

8. M.A. Sheremet, T.A. Trifonova, Transport Porous Media 101 (2014).

9. I.A. Aleshkova, M.A. Sheremet, Int. J. Heat Mass Transfer 53 (2010). 